\title{
Liraglutide in people treated for type 2 diabetes with multiple daily insulin injections: randomised clinical trial (MDI Liraglutide trial)
}

\author{
Marcus Lind, ${ }^{1,2}$ Irl B Hirsch, ${ }^{3}$ Jaakko Tuomilehto, ${ }^{4}$ Sofia Dahlqvist, ${ }^{2}$ Bo Ahrén, ${ }^{5}$ Ole Torffvit, ${ }^{5}$ \\ Stig Attvall, ${ }^{1}$ Magnus Ekelund, ${ }^{5}$ Karin Filipsson, ${ }^{5}$ Bengt-Olov Tengmark, ${ }^{6}$ Stefan Sjöberg, ${ }^{7}$ \\ Nils-Gunnar Pehrsson ${ }^{8}$
}

1'nstitute of Medicine, University of Gothenburg, Gothenburg, Sweden

2Department of Medicine, Uddevalla Hospital, NU Hospital Group, 45180 Uddevalla,

Sweden

University of Washington, Seattle, WA, US

${ }^{4}$ Centre for Vascular Prevention, Danube-University, Krems, Austria; Department of Chronic Disease Prevention, National Institute for Health and Welfare, Helsinki, Finland; Diabetes Research Group, King Abdulaziz University, Jeddah, Saudi Arabia

${ }^{5}$ Lund University, Lund, Sweden ${ }^{6}$ Citydiabetes, Stockholm, Sweden

${ }^{7}$ Karolinska Institutet, Karolinska University Hospital Huddinge,

Stockholm, Sweden

Department of Medicine,

Halland County Hospital,

Halmstad, Sweden

8Statistiska Konsultgruppen, Gothenburg, Sweden

Correspondence to: M Lind

lind.marcus@telia.com

Additional material is published online only. To view please visit the journal online (http://dx.doi. org/10.1136/bmj.h5364)

Cite this as: BMJ 2015;351:h5364 doi: $10.1136 /$ bmj.h5364

Accepted: 20 September 2015

\section{ABSTRACT}

\section{STUDY QUESTION}

What are the effects of liraglutide, an incretin based treatment, on glycaemic control in people with type 2 diabetes treated with multiple daily insulin injections?

\section{METHODS}

The study was a randomised, double blind, placebo controlled trial with a parallel group design carried out at 13 hospital based outpatient clinics and one primary care unit in Sweden. Patients were considered eligible for inclusion if they had type 2 diabetes and inadequate glycaemic control ( $\mathrm{HbA} 1 \mathrm{c}$ concentrations $\geq 58 \mathrm{mmol} / \mathrm{mol}(7.5 \%)$ and $\leq 102 \mathrm{mmol} / \mathrm{mol}(11.5 \%)$ ), a body mass index of $27.5-45 \mathrm{~kg} / \mathrm{m}^{2}$, and required multiple daily insulin injections. Overall, 124 participants were randomised 1:1 to subcutaneous liraglutide or placebo by minimisation allocation. The main outcome measure was change in $\mathrm{HbA1c}$ level from baseline to week 24 .

\section{STUDY ANSWER AND LIMITATIONS}

Liraglutide was associated with a significant reduction of $16.9 \mathrm{mmol} / \mathrm{mol}(1.5 \%)$ in $\mathrm{HbA1c}$ versus $4.6 \mathrm{mmol} /$ $\mathrm{mol}(0.4 \%)$ for placebo, difference $-12.3 \mathrm{mmol} / \mathrm{mol}$ ( $95 \%$ confidence interval -15.8 to $-8.8 \mathrm{mmol} / \mathrm{mol}$; $-1.13 \%,-1.45$ to $-0.81 \mathrm{mmol} / \mathrm{mol})$. Body weight was significantly reduced in participants in the liraglutide compared with placebo group $(3.8 v 0.0 \mathrm{~kg}$, difference $-3.8,-4.9$ to $-2.8 \mathrm{~kg}$ ), and total daily insulin doses were significantly reduced, by 18.1 units and 2.3 units (difference $-15.8,-23.1$ to -8.5 units). Reductions in mean and standard deviation of glucose levels estimated by masked continuous glucose monitoring were significantly greater in the liraglutide group than placebo group ( -1.9 and $-0.5 \mathrm{mmol} / \mathrm{L})$. Neither group experienced severe hypoglycaemic events nor were there any significant differences in symptomatic or asymptomatic non-severe hypoglycaemia $(<4.0$ or $<3.0 \mathrm{mmol} / \mathrm{L}$ ). The mean number of non-severe

\section{WHAT IS ALREADY KNOWN ON THIS TOPIC}

Earlier studies showed the efficiency of incretin based treatments in the early stages of type 2 diabetes and when added to oral antidiabetic drugs or basal insulin only-that is, not multiple daily insulin injections

\section{WHAT THIS STUDY ADDS}

Adding liraglutide to multiple daily insulin injections at late stages of type 2 diabetes is associated with improved glycaemic control, reduced body weight and lower total daily insulin doses

Masked continuous glucose monitoring was performed during the trial to obtain estimates of glucose levels every minute, which showed that glycaemic variability was reduced in patients treated with liraglutide

symptomatic hypoglycaemic events ( $<4.0 \mathrm{mmol} / \mathrm{L})$ during follow-up was 1.29 in the liraglutide group and 1.24 in the placebo group $(P=0.96)$. One of the study's limitations was its relatively short duration. Sustained effects of liraglutide have, however, been found over lengthier periods in connection with other treatment regimens. Cardiovascular safety and potential adverse events during longer exposure to liraglutide need to be evaluated. Nausea was experienced by 21 (32.8\%) participants in the liraglutide group and $5(7.8 \%)$ in the placebo group and $3(5 \%)$ and 4 (7\%) participants in these groups, respectively, had any serious adverse event.

\section{WHAT THIS STUDY ADDS}

Adding liraglutide to multiple daily insulin injections in people with type 2 diabetes improves glycaemic control without an increased risk of hypoglycaemia, reduces body weight, and enables patients to lower their insulin doses.

FUNDING, COMPETING INTERESTS, DATA SHARING This study was an investigator initiated trial, supported in part by Novo Nordisk and InfuCare. Potential competing interests have been reported and are available on thebmj.com.

\section{STUDY REGISTRATION}

EudraCT 2012-001941-42.

\section{Introduction}

Good glycaemic control is a cornerstone of the prevention of complications among people with type 2 diabetes. ${ }^{1-3}$ To obtain good glycaemic control, people with type 2 diabetes are generally treated with metformin and diet as the first line treatment. ${ }^{4-6}$ Sulphonylureas have traditionally been recommended as second line treatment. ${ }^{4-7}$ In recent years, however, consensus guidelines from the American Diabetes Association and European Association for the Study of Diabetes have focused on an individualised perspective in the choice of non-insulin glucose lowering drugs after metformin. ${ }^{6}$ Sulphonylureas, incretin based treatments, glitazones, sodium-glucose cotransporter 2 inhibitors, and basal insulin are all potential treatment options where individualised needs should be considered for the effects on body weight, hypoglycaemia, glycated haemoglobin (HbA1c), and costs. ${ }^{68}$

When insulin treatment is initiated, it usually includes a basal or premixed formulation. ${ }^{6}$ As a final step, multiple daily insulin injections with basal and prandial insulin have been standard when glycaemic control does not meet targets. ${ }^{6}$ Obesity is another comorbid condition in most people with type 2 diabetes, ${ }^{9}$ 
and adding basal insulin treatment is associated with further weight gain and an increased risk of hypoglycaemia. ${ }^{16}$ Multiple daily insulin injections generally result in even greater weight gain. Hence in many people multiple daily insulin injections result in even greater insulin resistance and obesity, and patients often still do not reach adequate glycaemic control. ${ }^{10}$

To determine whether people with type 2 diabetes treated with multiple daily insulin injections may benefit from adjunctive treatment using novel glucose lowering agents, we evaluated the effects of the incretin based treatment liraglutide.

\section{Methods}

The study design and predefined endpoints are described in detail elsewhere. ${ }^{11}$ Briefly, the study was a randomised, double blind, placebo controlled trial with a parallel group design conducted at 14 sites (13 hospital based outpatient clinics and one primary care unit) in Sweden. Written and verbal informed consent was obtained from all participants. The inclusion criteria were people with type 2 diabetes treated with multiple daily insulin injections, HbA1c concentrations $\geq 58$ $\mathrm{mmol} / \mathrm{mol}(7.5 \%)$ and $\leq 102 \mathrm{mmol} / \mathrm{mol}(11.5 \%)$, and a body mass index of $27.5-45 \mathrm{~kg} / \mathrm{m}^{2}$. Multiple daily insulin injections were defined as separate basal and mealtime insulin components, including at least two daily mealtime insulin doses. We excluded people using premixed insulin. Participants were required to have a fasting C peptide level of $0.1 \mathrm{nmol} / \mathrm{L}$ or higher. Some of the inclusion and exclusion criteria were subject to minor changes during the study, partly to enable the use of more general and conventional cut-offs for variables and partly to facilitate further recruitment. During the initial part of the study we changed the lower cut-off for $\mathrm{HbA1c}$ from $64 \mathrm{mmol} / \mathrm{mol}(8.0 \%)$ to $58 \mathrm{mmol} / \mathrm{mol}(7.5 \%)$ and body mass index from 28.0 to $27.5 \mathrm{~kg} / \mathrm{m}^{2}$. We also changed the cut-offs for $\mathrm{C}$ peptide, calcitonin, fasting glucose, creatinine, and age during the study, and clarified contraceptive methods and use of glucocorticoids (see supplementary file for details). Other inclusion and exclusion criteria have been described previously. ${ }^{11}$

After a maximum run-in period of eight weeks, we randomised participants to either subcutaneous liraglutide or placebo. The composition of the placebo was the same as for liraglutide but with the absence of the active pharmaceutical ingredient. The study was double blinded and we used minimisation allocation to randomise participants 1:1 to liraglutide or placebo (see supplementary file for details). Using the Dexcom G4 PLATINUM (San Diego, CA) continuous glucose monitoring system, we carried out masked continuous glucose monitoring during one week of the eight week run-in period, week 12 of the trial, and one of the two final weeks of the follow-up period of 24 weeks. The device used for continuous glucose monitoring consists of a subcutaneous sensor, a wireless transmitter, and a receiver. ${ }^{12}$ The sensor continuously measures the glucose values in interstitial fluid over one week and sends data to the receiver. During masking the receiver does not display the values but rather stores them for downloading. We measured capillary glucose values before meals and 1.5 hours after meals during at least two days before baseline and at the end of follow-up. During the study we used the Contour XT blood glucose meter (Bayer) to measure capillary glucose levels. We used the diabetes treatment satisfaction questionnaire to estimate patient satisfaction with treatment. This validated questionnaire consists of eight questions and has been used in many clinical trials of diabetes treatment. Two versions are used, one for patients to record current satisfaction with treatment and one for patients to retrospectively compare satisfaction before and after study treatment. At each visit we asked participants to report any potential adverse events using an open ended question.

Liraglutide or placebo was administered at a dose of 0.6 mg during week $1,1.2 \mathrm{mg}$ during week 2 , and $1.8 \mathrm{mg}$ during week 3 and onwards. The patients chose when to administer the drug during the day, and they were supposed to use the same timing each day during the trial. The periods for increase of dose were extended based on individual tolerance to the trial product. During the trial, the highest tolerated dose of liraglutide or placebo was used.

Since the participants had inadequate glycaemic control at inclusion (HbA1c $\geq 58 \mathrm{mmol} / \mathrm{mol}(7.5 \%)$ ), no general reduction in insulin doses were recommended when initiating or titrating liraglutide or placebo. ${ }^{11}$ Reductions in insulin were only considered in participants with normal or close to normal glucose levels at fasting or before meals $(<7.0 \mathrm{mmol} / \mathrm{L}(126 \mathrm{mg} / \mathrm{dL}))$ based on self measured blood glucose levels (7 point profile) for two days.

If glucose levels were not on target after titrating liraglutide or placebo we recommended increasing insulin doses to original levels. After the titration phase of liraglutide and placebo, we advised the participants to adjust their insulin doses throughout the study as performed daily before enrolling in the trial. The participants were advised to measure three or four glucose values each day before meals and at bedtime according to clinical guidelines for potentially adjusting insulin doses, diet, or physical activity.

After randomisation, follow-up visits took place at weeks 6, 12, 18, and 24. At all visits we checked HbA1c levels, insulin doses, hypoglycaemic events, adverse events, blood pressure, and weight. Additionally, extensive clinical examinations, assessment of treatment satisfaction, and laboratory testing, including of biobank samples, were carried out at weeks 12 and $24 .{ }^{11}$

If fasting self measured blood glucose values taken on three separate days or any fasting plasma glucose samples analysed by the central laboratory exceeded 15.5 $\mathrm{mmol} / \mathrm{L}(279 \mathrm{mg} / \mathrm{dL})$ from baseline to week 12, or 13.5 $\mathrm{mmol} / \mathrm{L}$ ( $245 \mathrm{mg} / \mathrm{dL}$ ) from week 12 to week 24 , the participant was contacted for an unscheduled visit about rescue treatment. If an increased fasting plasma glucose level was confirmed by the central laboratory and no treatable intercurrent cause for hyperglycaemia was diagnosed, rescue treatment was initiated, which primarily consisted of the investigator assisting in increasing insulin doses.

According to guidelines for trials we defined hypoglycaemias as non-severe symptomatic, non-severe asymptomatic, and severe requiring the assistance of 
another person. ${ }^{13}$ The number of non-severe hypoglycaemias were predefined to be evaluated both below 4.0 $\mathrm{mmol} / \mathrm{L}\left(72 \mathrm{mg} / \mathrm{dL}\right.$ ) and $3.0 \mathrm{mmol} / \mathrm{L}(54 \mathrm{mg} / \mathrm{dL}) .{ }^{11}$ The participants were counselled on typical symptoms of hypoglycaemia and provided with a diabetes diary to record glucose values, signs of hypoglycaemia, and recovery from symptoms after intake of carbohydrates. If symptoms occurred we instructed the participants to perform a finger stick glucose measurement immediately, but to avoid delay in treating symptoms.

The primary endpoint was change in HbA1c level between baseline and week 24 . Novo Nordisk provided the study drug and treatment codes. Apoteket (National Pharmacy), Sweden, handled study treatment logistics. Gothia Forum (Gothenburg, Sweden) monitored the study. Laboratory tests were measured at the Research Centre for Laboratory Medicine at Karolinska University Hospital, Stockholm, Sweden.

\section{Patient involvement}

No patients were involved in setting the research question or the outcome measures, nor were they involved in recruitment or the design and implementation of the study. There are no plans to involve patients in dissemination of the results.

\section{Statistical analysis}

The main statistical analyses for predefined endpoints were presented in the original protocol and are published elsewhere. ${ }^{11} \mathrm{~A}$ detailed statistical analysis plan was signed before the database was locked. The full analysis set, used for the primary efficacy analysis, consisted of all randomised participants who received at least one dose of study drug and had at least one follow-up measurement. The per protocol population consisted of all participants who had at least one visit at week 18 or 24 during follow-up, no major protocol deviations, and was determined before the database was locked. The primary efficacy analysis was the change in

\begin{tabular}{|c|c|}
\hline \multicolumn{2}{|c|}{ Assessed for eligibility $(n=180)$} \\
\hline & $\begin{array}{l}\text { Excluded }(n=56) \text { : } \\
\quad \text { Not meeting inclusion criteria }(n=51) \\
\text { Refused to participate }(n=5)\end{array}$ \\
\hline & \\
\hline \multicolumn{2}{|c|}{ Randomised $(\mathrm{n}=124)$} \\
\hline$\downarrow$ & 7 \\
\hline $\begin{array}{l}\text { Assigned to receive intervention liraglutide } \\
(n=64) \text { : } \\
\text { Received intervention as assigned }(n=64) \\
\text { Did not receive assigned intervention }(n=0)\end{array}$ & $\begin{array}{l}\text { Assigned to receive intervention placebo } \\
(n=60) \text { : } \\
\text { Received intervention as assigned }(n=60) \\
\text { Did not receive assigned intervention }(n=0)\end{array}$ \\
\hline$\downarrow$ & $\downarrow$ \\
\hline $\begin{array}{l}\text { Lost to follow-up (did not come to scheduled } \\
\text { visits) }(n=1) \\
\text { Discontinued intervention (both had } \\
\text { gastrointestinal symptoms) }(n=2)\end{array}$ & $\begin{array}{l}\text { Lost to follow-up (did not come to scheduled } \\
\text { visits) }(n=1) \\
\text { Discontinued intervention (safety reasons) } \\
(n=2)\end{array}$ \\
\hline$\downarrow$ & $\downarrow$ \\
\hline $\begin{array}{l}\text { Included in full analysis set }(n=63) \\
\text { Excluded from full analysis set (no follow-up } \\
\text { measurement) }(n=1)\end{array}$ & $\begin{array}{l}\text { Included in full analysis set }(n=59) \\
\text { Excluded from full analysis set (no follow-up } \\
\text { measurement) }(n=1)\end{array}$ \\
\hline
\end{tabular}

Fig 1 | Flow of participants through trial
HbA1c level from baseline to 24 weeks between the two treatment groups using analysis of covariance, with HbA1c at baseline as the covariate on the full analysis set. The last observation was carried forward from six weeks. In all efficacy analyses we excluded measurements obtained after rescue treatment.

For comparison between the two study groups we used Fisher's exact test for dichotomous variables, Mantel-Haenszel's $\chi^{2}$ test for ordered categorical variables, and Fisher's non-parametric permutation test for continuous baseline variables. For efficacy variables of change between baseline and 24 weeks, we used analysis of covariance with baseline value as a covariate. A sensitivity analysis was performed on all predefined endpoints, including all randomised participants irrespective of whether they took the study drugs or had any valid follow-up measurements. The methodology was the same as for the primary efficacy analysis except that we used the last observation carried forward principle from baseline values onwards. All significance tests were two sided and conducted at the 5\% significance level. For all analyses we used SAS System version 9 (SAS Institute, Cary, NC).

The study was powered to detect a difference in HbA1c concentration of $7 \mathrm{mmol} / \mathrm{mol}(0.7 \%)$ between liraglutide and placebo. For both groups we assumed a standard deviation of $1.2 \%$ for a change in HbA1c level, ${ }^{14}$ thus we needed 57 participants in each group. We assumed a drop-out rate of 5\%, thus we needed 60 participants in each group.

\section{Results}

Enrolment occurred between February 2013 and February 2014, and the last participant completed the trial in August 2014. Figure 1 shows the flow of participants through the trial, including those who were lost to follow-up and discontinued the trial.

\section{Full analysis set evaluation}

In total 124 participants were randomised and 122 had at least one follow-up measurement and were included in the full analysis set used for the primary efficacy analysis. Table 1 shows the baseline characteristics of the participants. The HbA1c levels in the full analysis set were $74.6 \mathrm{mmol} / \mathrm{mol}(8.98 \%)$ for participants in the liraglutide group and $74.4 \mathrm{mmol} / \mathrm{mol}(8.96 \%)$ for those in the placebo group. Age, sex, body mass index , total daily insulin dose, number of insulin injections, duration of diabetes, and other participant characteristics were similar at baseline between the groups.

Table 2 shows the results of the predefined endpoints in the evaluation of the full analysis set. The HbA1c concentration was reduced by $16.9 \mathrm{mmol} / \mathrm{mol}(1.54 \%)$ in the liraglutide group compared with $4.57 \mathrm{mmol} / \mathrm{mol}$ $(0.42 \%)$ in the placebo group (difference $-12.3 \mathrm{mmol} /$ mol, 95\% confidence interval -15.8 to -8.8 and $-1.13 \%$, -1.45 to -0.81 ; $\mathrm{P}<0.001)$. More participants in the liraglutide group (42.9\%) than in the placebo group $(5.1 \%)$ reached an $\mathrm{HbA1c}$ concentration $<53 \mathrm{mmol} / \mathrm{mol}(7.0 \%$; $\mathrm{P}<0.001)$. A greater proportion of participants treated with liraglutide also reached HbA1c concentrations $<58$ $\mathrm{mmol} / \mathrm{mol}(7.5 \%)$ and $64 \mathrm{mmol} / \mathrm{mol}$ (8.0\%, table 2). 


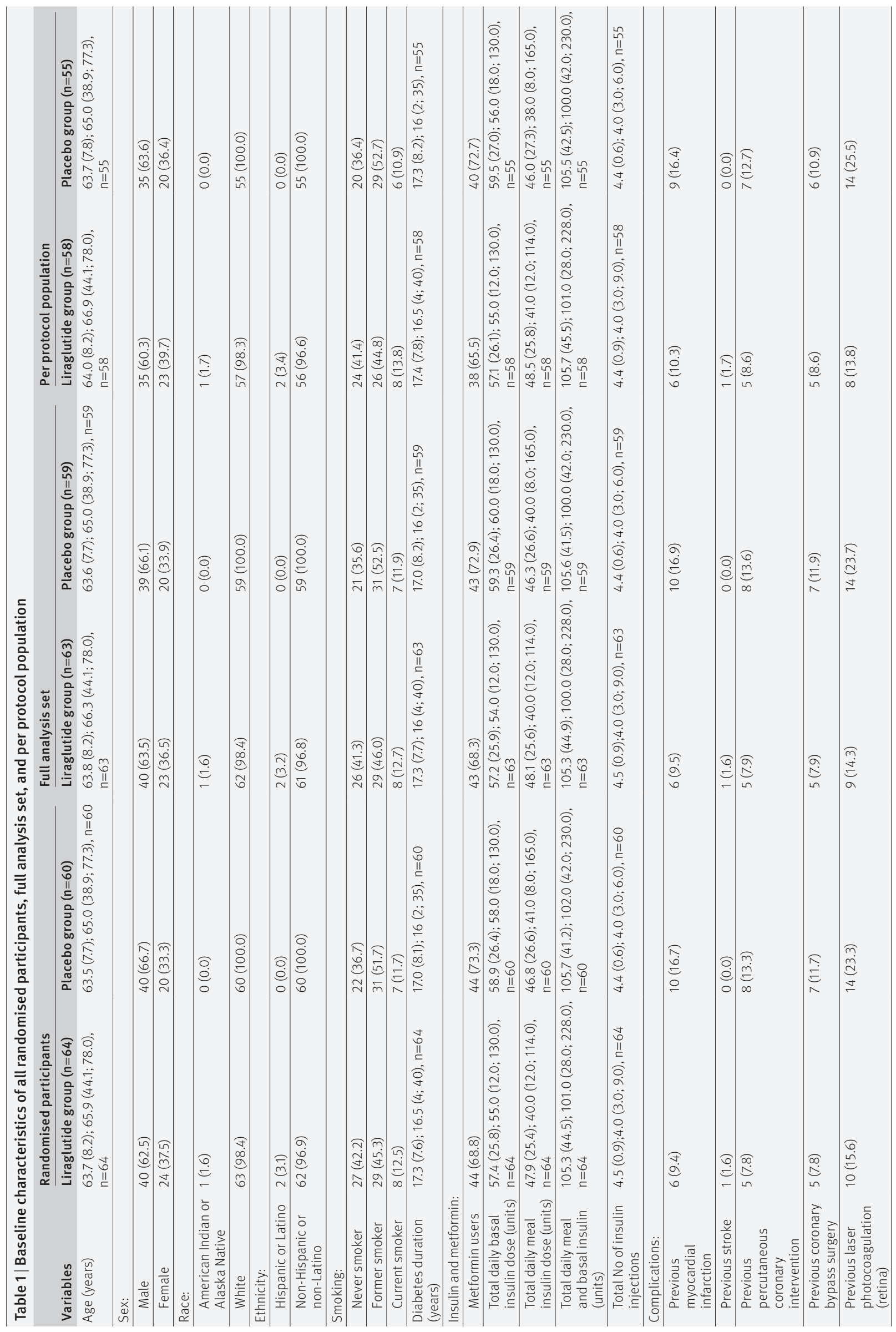

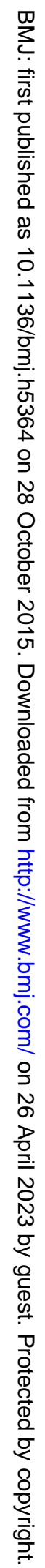




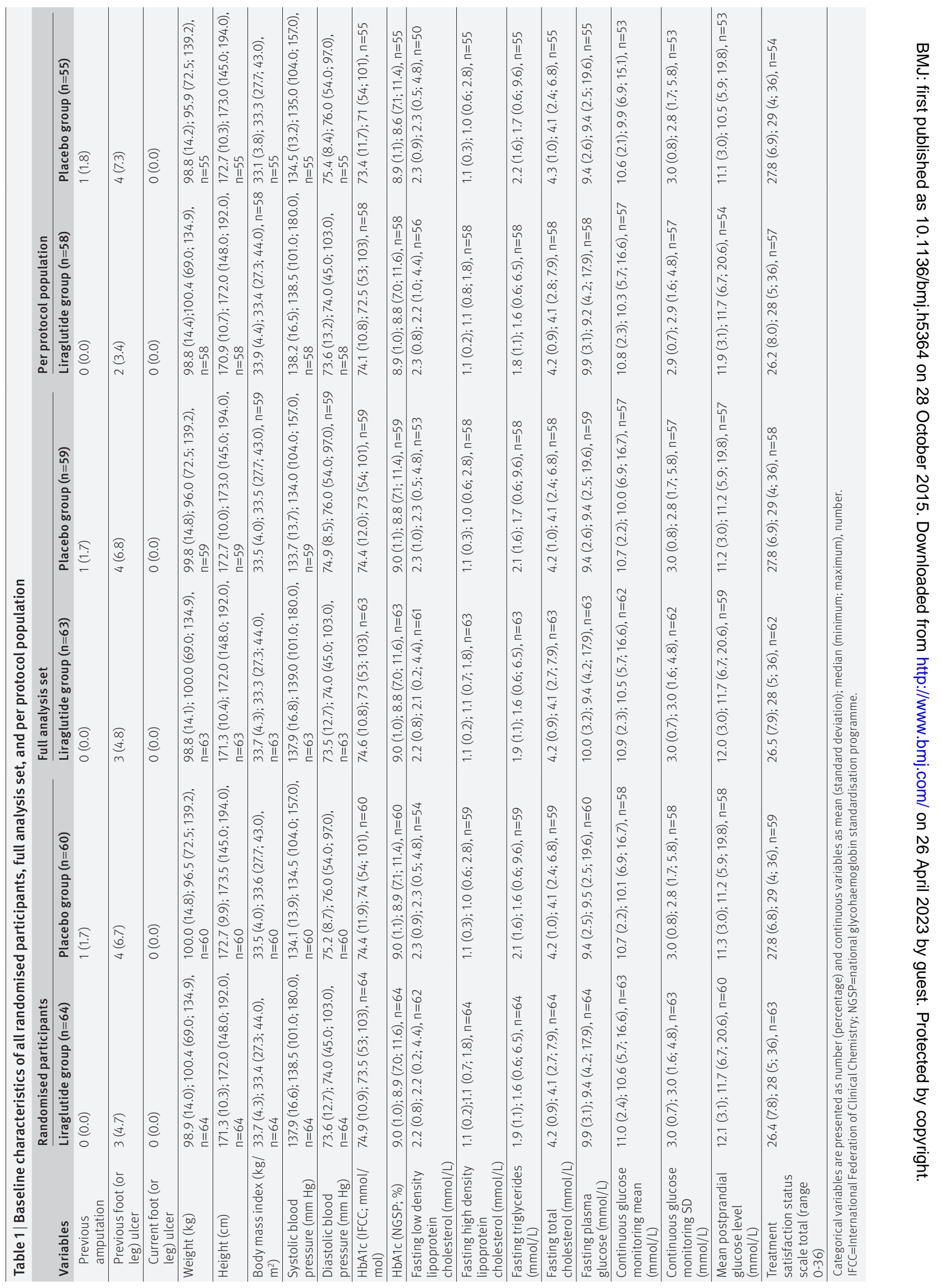



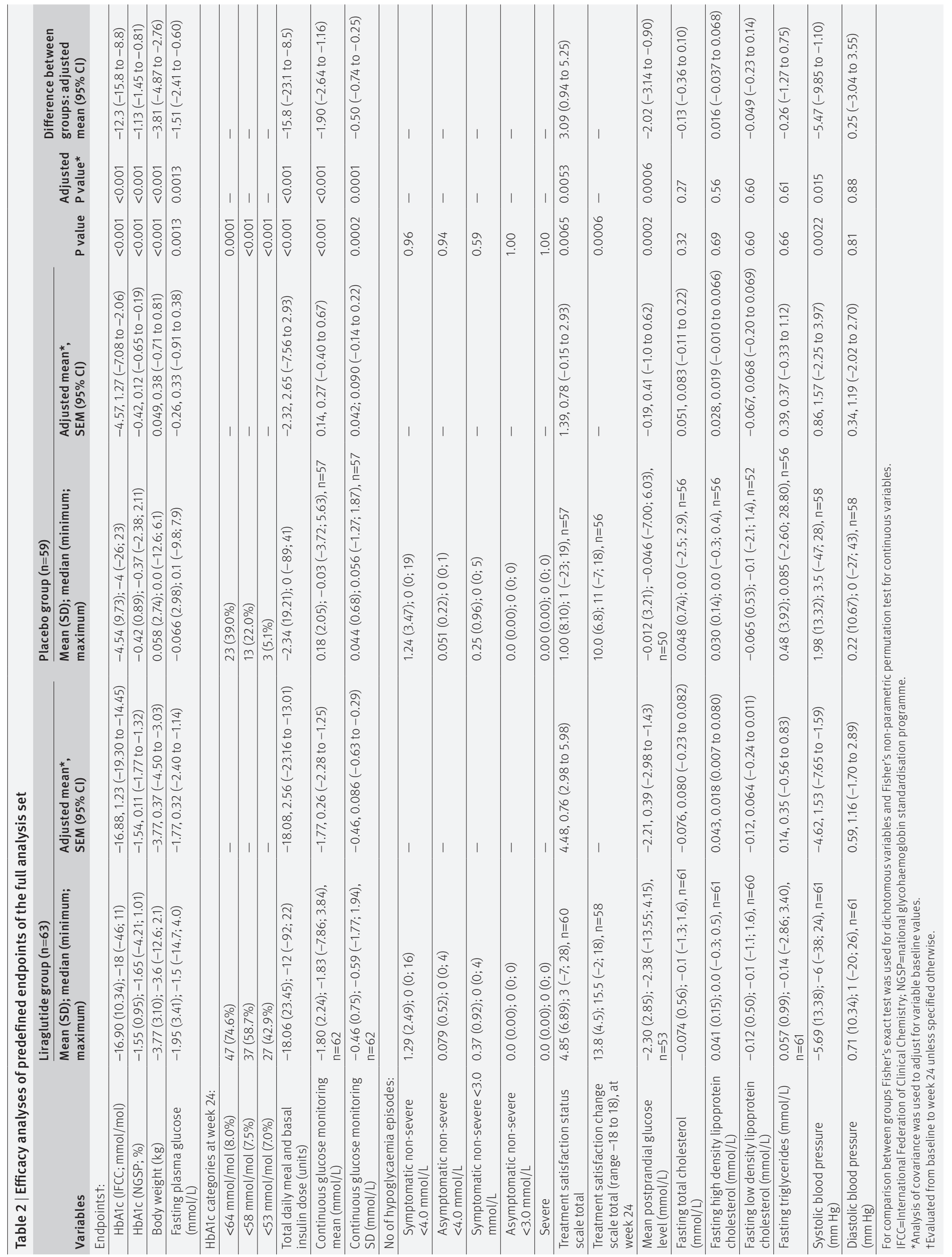
Body weight was reduced in participants treated with liraglutide, by $3.8 \mathrm{~kg}$, but not in participants treated with placebo $(0.0 \mathrm{~kg}$, difference $-3.8 \mathrm{~kg}, 95 \%$ confidence interval -4.9 to $-2.8 ; \mathrm{P}<0.001)$. The daily insulin dose was reduced by 18.1 units with liraglutide and by 2.3 units with placebo (difference -15.8 units, 95\% confidence interval -23.1 to $-8.5 ; \mathrm{P}<0.001$ ). In the liraglutide group the total daily basal insulin dose was reduced by 6.8 units and total daily mealtime insulin dose by 11.2 units. In the placebo group the corresponding reductions were 0.5 units and 1.9 units.

Reductions in fasting and postprandial glucose levels were greater in the liraglutide group than in the placebo group (difference -1.5 and $-2.0 \mathrm{mmol} / \mathrm{L}$ ( -27 and -36 $\mathrm{mg} / \mathrm{dL}$ ), respectively; $\mathrm{P}<0.01$ for both). Reductions in mean glucose levels and standard deviation estimated by masked continuous glucose monitoring were significantly greater in the liraglutide group than in the placebo group ( -1.9 and $-0.5 \mathrm{mmol} / \mathrm{L}(-34.2$ and $-9 \mathrm{mg} /$ $\mathrm{dL}$ ), respectively; $\mathrm{P}<0.001)$.

Neither group experienced severe hypoglycaemic events and no significant differences were found in symptomatic or asymptomatic non-severe hypoglycaemias, with values $<4.0$ or $<3.0 \mathrm{mmol} / \mathrm{L}$ ( $<72$ or $<54 \mathrm{mg} /$ $\mathrm{dL}$ ) between the liraglutide and placebo groups (table 2). Non-severe symptomatic hypoglycaemia $<4.0 \mathrm{mmol} / \mathrm{L}$ was most common, with a mean of 1.29 events during follow-up in the liraglutide group and 1.24 in the placebo group $(\mathrm{P}=0.96)$.

Treatment satisfaction was significantly greater with liraglutide than with placebo, when estimated by both of the diabetes treatment satisfaction questionnaires (current and retrospective) (table 2).

Systolic blood pressure decreased by $4.62 \mathrm{~mm} \mathrm{Hg}$ in participants treated with liraglutide and increased by $0.86 \mathrm{~mm} \mathrm{Hg}$ in participants treated with placebo (difference $-5.47 \mathrm{~mm} \mathrm{Hg}, 95 \%$ confidence interval -9.85 to $-1.10, \mathrm{P}=0.015)$. No significant difference was found for diastolic blood pressure, as was the case for levels of total cholesterol, low density lipoprotein cholesterol, high density lipoprotein, and triglycerides (table 2).

Of the 64 participants in the liraglutide group, 57 $(89.1 \%)$ received a dose of $1.8 \mathrm{mg}, 3(4.7 \%) 1.2 \mathrm{mg}$, and 4 (6.3\%) $0.6 \mathrm{mg}$ at the end of follow-up, whereas all 60 participants in the placebo group received $1.8 \mathrm{mg}$.

\section{Withdrawals}

Five participants withdrew overall: three in the liraglutide group and two in the placebo group.

\section{Rescue treatment}

One participant $(1.6 \%)$ in the liraglutide group and three $(5 \%)$ in the placebo group received rescue treatment. Rescue treatment was initiated during the first 12 weeks in two participants in the placebo group and during the second half of the trial in one participant in each group.

\section{Per protocol evaluations}

Overall, 58 (90.6\%) participants in the liraglutide group and $55(91.7 \%)$ in the placebo group were included in the predefined per protocol population. Supplementary etable 1 shows the reasons for excluding participants from the per protocol analysis. The difference in the change in HbA1c concentrations from baseline to 24 weeks between groups in favour of liraglutide was -12.4 $\mathrm{mmol} / \mathrm{mol}$ (95\% confidence interval -16.0 to -8.8 and $-1.14 \%,-1.47$ to $-0.80 ; \mathrm{P}<0.001)$. The same endpoints as in the intention to treat analysis became significant in favour of liraglutide in the per protocol analysis (see supplementary etable 2 ).

Time evaluations of change in HbA1c levels, weight, and total daily insulin dose

Figure 2 shows the change in HbA1c levels, weight, and total daily insulin dose for participants in the liraglutide and placebo groups at baseline and every six weeks. There was a significantly greater reduction in HbA1c concentration ( $-9.4 \mathrm{mmol} / \mathrm{mol}, 0.86 \%)$, weight $(-2.4 \mathrm{~kg})$, and insulin dose (-15.1 units) already at six weeks $(\mathrm{P}<0.001$ for all $)$ in participants treated with liraglutide compared with placebo.
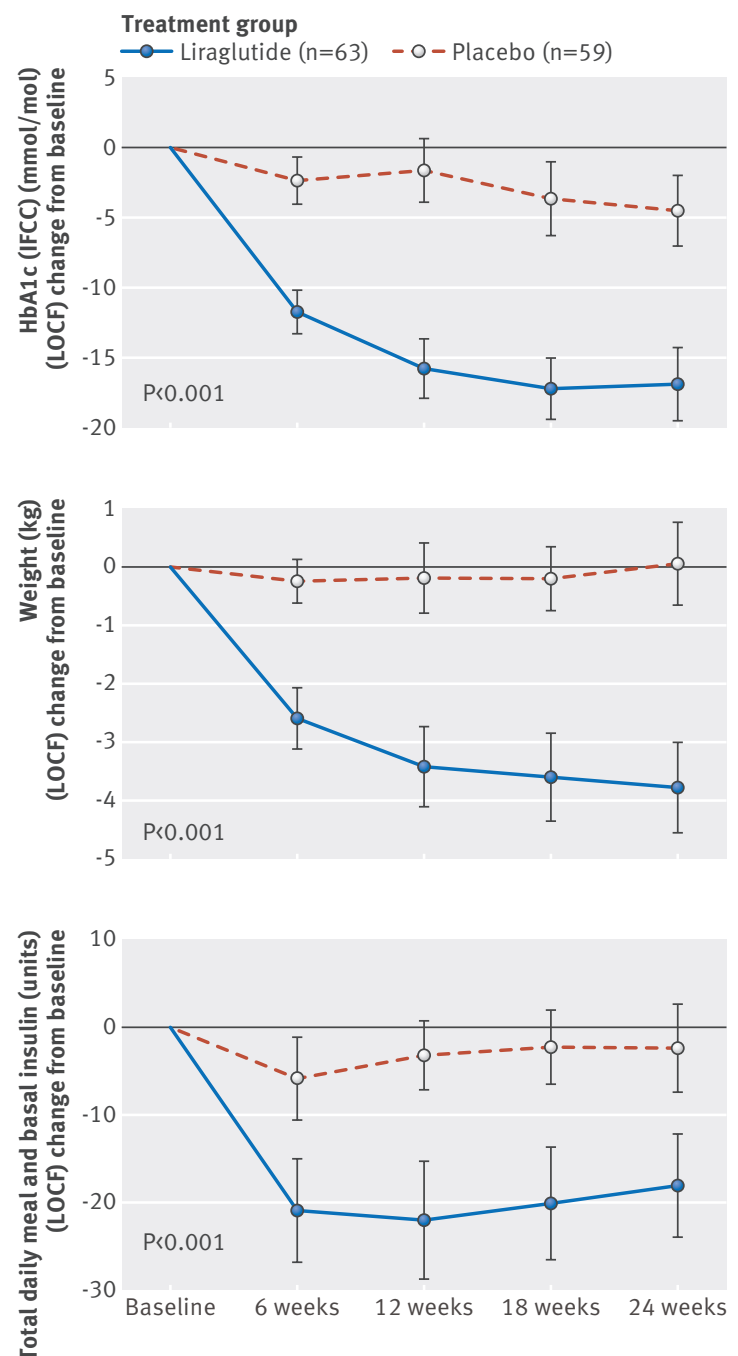

Fig 2 Change in HbA1c concentration, weight, and daily insulin dose by treatment group over time (mean and $95 \%$ confidence interval). IFCC= International Federation of Clinical Chemistry; LOCF=last observation carried forward 


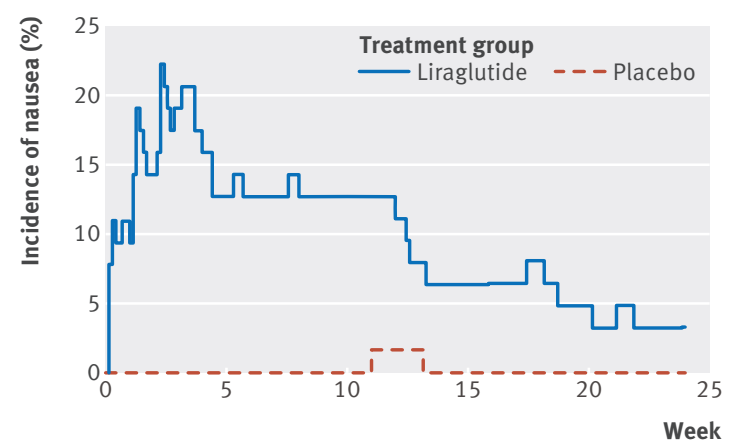

Fig 3 | Incidence of nausea (\%) by treatment group over time (safety population)

\section{Adverse events}

During follow-up, gastrointestinal symptoms were experienced by $30(46.9 \%)$ participants in the liraglutide group and eight (13.3\%) in the placebo group. The corresponding numbers for nausea were $21(32.8 \%)$ and $1(1.7 \%)$ and for diarrhoea were $5(7.8 \%)$ and $3(5.0 \%)$. Gastrointestinal symptoms were mainly present at the start of the trial and at 24 weeks. Only two (3\%) participants in the liraglutide group had nausea (fig 3). Overall, three (4.7\%) participants in the liraglutide group and four (6.7\%) in the placebo group had any serious adverse event (table 3 ).

\section{Sensitivity analysis}

We performed a sensitivity analysis on all randomised participants $(n=124)$-that is, also including the two participants without any valid follow-up measurements who were not included in the primary efficacy analysis (full analysis set evaluation). All predefined endpoints were evaluated (see supplementary etable 3). The results remained unchanged; all endpoints that were highly significant in favour of liraglutide in the primary efficacy analysis remained so, with a greater reduction in HbA1c concentrations of $12.1 \mathrm{mmol} / \mathrm{mol}(1.1 \%)$, compared with participants in the placebo group.

\section{Discussion}

In this double blind randomised trial of incretin based treatment added to multiple daily insulin injections for people with type 2 diabetes, glycaemic control was significantly improved with the addition of liraglutide. Compared with controls, the HbA1c level was reduced by $12.3 \mathrm{mmol} / \mathrm{mol}$ (1.1\%). Moreover, weight was reduced by $3.8 \mathrm{~kg}$ in participants treated with liraglutide compared

\begin{tabular}{|c|c|c|c|c|}
\hline \multirow[b]{2}{*}{ Serious adverse events } & \multicolumn{2}{|c|}{ Liraglutide group $(n=64)$} & \multicolumn{2}{|c|}{ Placebo group $(n=60)$} \\
\hline & Events & No (\%) with events & Events & No (\%) with events \\
\hline Any serious adverse event: & 3 & $3(5)$ & 8 & $4(7)$ \\
\hline \multicolumn{5}{|l|}{ Adverse events: } \\
\hline Atrial fibrillation & - & - & 3 & $1(2)$ \\
\hline Cardiac failure & - & - & 1 & $1(2)$ \\
\hline Vitreous detachment & - & - & 1 & $1(2)$ \\
\hline Generalised oedema & - & - & 1 & $1(2)$ \\
\hline Cholecystitis & - & - & 1 & $1(2)$ \\
\hline Hip surgery & 1 & $1(2)$ & - & - \\
\hline Admission to hospital & 2 & $2(3)$ & 1 & $1(2)$ \\
\hline
\end{tabular}

with controls. Improved glycaemic control was obtained despite a lowering of insulin doses by 15.8 units in the liraglutide group compared with controls and without any increased risk of hypoglycaemia, perhaps owing to lower glycaemic variability as confirmed by masked continuous glucose monitoring. Participants treated with liraglutide experienced significantly more gastrointestinal side effects than controls, although these events were mostly confined to the beginning of the trial.

\section{Comparison with other studies}

In previous blinded randomised controlled trials where the glucagon-like peptide 1 analogues lixisenatide or exenatide DPP4 (dipeptidyl peptidase-4) inhibitors were added to insulin treatment, mostly basal, the absolute reduction in $\mathrm{HbA1c}$ level was around $5 \mathrm{mmol} / \mathrm{mol}(0.5 \%)$ greater in the treatment group than in the controls. ${ }^{15}$

When DPP4 inhibitors have been added to current insulin treatment, body weight has not been reduced, and insulin doses were marginally reduced by 0-4 units. ${ }^{16-19}$ Adding exenatide to insulin glargine led to a $2.8 \mathrm{~kg}$ weight reduction, an insulin dose 7 units lower, and a $7.7 \mathrm{mmol} /$ mol $(0.70 \%)$ reduction in HbA1c level. ${ }^{20}$ Adding lixisenatide was associated with $1.3 \mathrm{~kg}$ and $0.9 \mathrm{~kg}$ weight reductions, $4 \mathrm{mmol} / \mathrm{mol}(0.4 \%)$ and $3 \mathrm{mmol} / \mathrm{mol}(0.3 \%)$ reductions in $\mathrm{HbA1c}$ level, and reductions in insulin by 3.7 units and 2.2 units in two separate studies, respectively, when added to insulin treatment. ${ }^{2122}$ Liraglutide and albiglutide have been evaluated in non-blinded trials as add-on treatment to basal insulin. ${ }^{2324}$ Today, glucagon-like peptide 1 analogues are viewed as a treatment option in addition to basal insulin. ${ }^{8}$ Liraglutide has shown superior effects over other incretin based treatments in populations treated with oral glucose lowering drugs. ${ }^{25-27}$

\section{Explanations and implications}

Treatment with multiple daily insulin injections in people with type 2 diabetes has been associated with lower insulin production compared with people using other treatment regimens. ${ }^{28}$ Lower insulin production has also been associated with a longer duration of diabetes, ${ }^{29}$ as is the case for worse glycaemic control. ${ }^{730}$ The average duration of diabetes in the current trial was more than 17 years, considerably longer than in other trials of type 2 diabetes, ${ }^{114-27} 3031$ including those of gastric bypass surgery, where it was around eight years. ${ }^{32}$ When incretin based treatments were introduced they were initially thought to be efficient in people with early disease. ${ }^{33}$ The current findings complement earlier results and confirm that incretins are effective over the entire time continuum of type 2 diabetes. It is possible that besides liraglutide's mechanism of action, ${ }^{14}{ }^{15}$ including an insulinotrophic and glucagonostatic effect, weight reduction from liraglutide use may be partially responsible for an increased effect of exogenous insulin delivered by multiple daily insulin injections in these participants. It is also possible that glucagon-like peptide 1 receptor agonists may have a beneficial effect in people with type 1 diabetes, among others, through a glucagonostatic effect; this is being evaluated in ongoing randomised trials.

Masked continuous glucose monitoring has generally not been performed in blinded randomised trials of incretin 
based treatments. ${ }^{1415-27}$ Besides improvement in glycaemic control and weight reduction, participants experiencing large fluctuations in glucose levels could benefit from the addition of liraglutide to their treatment regimen. In our study, the standard deviation of glucose levels was significantly reduced after treatment with liraglutide. Systolic blood pressure was also reduced, by approximately $5 \mathrm{~mm}$ $\mathrm{Hg}$, which may also be a cardioprotective benefit. ${ }^{34}$

The current treatment algorithm was not associated with any severe adverse events or severe hypoglycaemias; overall the number of non-severe hypoglycaemias was low during the trial. Such a titration algorithm with a low risk of hypoglycaemia may be of general interest in clinical practice. In contrast with other studies, ${ }^{1516-22}$ based on two updated glucose profiles, people with glucose levels on target were advised to reduce insulin doses by 20-30\% when initiating or titrating liraglutide or placebo. Hence, those with poor glycaemic control could avoid unnecessary reductions in insulin, and those with relatively good glucose levels may be able to avoid hypoglycaemia. Although gastrointestinal side effects were more common in people treated with liraglutide in keeping with earlier trials, ${ }^{14}$ these usually appeared at the beginning of treatment and disappeared soon during the continuation of liraglutide, and resulted in only two participants discontinuing treatment.

\section{Strengths and limitations of this study}

A strength of the present study is the randomised blinded multicentre design. The trial was investigator initiated, and the manufacturer of liraglutide was not the sponsor of the trial, unlike earlier placebo controlled trials in the subject. ${ }^{145-22} \mathrm{~A}$ limitation is the relatively short duration of the study. However, we did not believe it to be ethical to extend the study duration because many participants had poor glycaemic control. Since the effect of liraglutide has been sustained over longer periods in other study settings, ${ }^{35}$ it is possible that it has longer durability in this patient population. Importantly, the aim of the current trial was to study the effects of adding liraglutide to multiple daily insulin injections and continuing regular treatment of people with type 2 diabetes. Hence insulin doses were not adjusted by investigators except for safety, although participants were instructed to continue to adjust doses. Thus the results of the present study may differ from those of a trial using a treat to target design, where insulin doses can be optimised by investigators simultaneous to adding liraglutide. Therefore our results may mimic the real world effects of liraglutide rather than a multiple intervention scenario consisting of optimising insulin doses and educating patients on titrating meal time insulin doses while initiating liraglutide.

Current trials of liraglutide are too short to asses overall cardiovascular risks and need to be evaluated in long term follow-up trials. ${ }^{36}{ }^{37}$ Recently, results from the long term trial with the glucagon-like peptide 1 analogue lixisenatide showed encouraging results on the cardiovascular safety of this agent. ${ }^{38}$ Heart rate was not recorded in the current trial. It is noteworthy, however, that glucagon-like peptide 1 analogues have been associated with a slight increase in heart rate. ${ }^{39}$ Possible risks of pancreatitis and pancreas and thyroidal medullary cancer associated with incretin based treatments have been discussed, ${ }^{40} 41$ but no causal association has been assessed and further evaluations are recommended in long term follow-up studies. ${ }^{39} 40$ In this study, there was no incidence of these events.

\section{Conclusions}

Liraglutide improves glycaemic control in people with type 2 diabetes treated with multiple daily insulin injections and long duration of disease. Liraglutide was also associated with reductions in body weight and insulin doses. Data on long term use of liraglutide regarding safety, such as risk of pancreatitis, cancer, and cardiovascular disease are needed.

We thank the participants, the staff at participating care units, the local laboratories assisting in taking blood samples and logistics of laboratory transports, and the central laboratory; Gothia Forum for monitoring the trial and Nordiclnfu Care for training regarding masked continuous glucose monitoring and support with continuous glucose monitoring receivers; and Novo Nordisk for support with liraglutide and placebo and partly financing the trial. We thank Joseph Murphy for editorial assistance with an earlier version of the manuscript.

Contributors: ML, IBH, and JT comprised the steering committee. ML and N-GP are the guarantors of the work and had full access to all the data in the study and takes responsibility for the integrity of the data and the accuracy of the data analysis. ML, IBH, JT, SD, and N-GP designed the statistical analysis plan together with Mattias Molin, Statistiska Konsultgruppen, Gothenburg, Sweden. All authors were involved in interpreting data and revising the first draft, which was written by ML. All authors approved the final draft the manuscript. The statistical analyses were performed by Mattias Molin, who did not want to participate as co-author since he wanted to remain independent of the trial and its results. Sponsor was the PI (first author) on behalf of the NU Hospital Group.

Funding: The current study was an investigator initiated trial, where Novo Nordisk provided financial support and study drugs but had no role in the design or execution of the trial; the interpretation, analysis, or publication of data; or the decision to submit the written report. NordicInfu Care provided continuous glucose monitoring systems and support.

Competing interests: All authors have completed the ICMJE uniform disclosure form at www.icmje.org/coi_disclosure.pdf (available on request from the corresponding author) and declare: ML and SD had support from Novo Nordisk for the submitted work; outside of the submitted work, authors have relations to the following companies that may take interest in the submitted work. ML has received research grants from AstraZeneca, Dexcom, and Novo Nordisk, been a consultant or received honorariums from Medtronic, Eli Lilly, Pfizer, Abbot Scandinavia, Bayer, Novo Nordisk, and Rubin Medical, and has participated in advisory boards for Novo Nordisk. IBH has received research grants from Sanofi-US, Novo Nordisk, and Halozyme, and has been a consultant for Abbott Diabetes Care, Valeritas, Roche Diagnostics, and Becton Dickinson. IT has received research support from Bayer, Boehringer Ingelheim, Merck Sharp \& Dohme, Novartis, Sanofi Aventis, and Servier, and has acted as a consultant, advisory board member, or speaker for Impeto Medical, Novartis, Novo Nordisk, Sanofi -Aventis, Eli Lilly, Merck Serono, Merck Sharp \& Dohme, Bayer, and Boehringer Ingelheim. BA has consulted for Novartis, GlaxoSmithKline, Merck, Sanofi, Novo Nordisk, Boehringer Ingelheim, and Takeda, and has received lecture fees from Novartis, Merck, Novo Nordisk, Sanofi, AstraZeneca, and GlaxoSmithKline. OT has been a consultant to Eli Lilly and Novo Nordisk. ME has been a consultant or received honorariums from Novartis, Merck Sharp \& Dohme, GlaxoSmithKline, Sanofi, Eli Lilly, and Rubin Medical, and has participated in advisory boards for Sanofi. KF has consulted for Novo Nordisk. BOT has participated in advisory boards for Novo Nordisk and Boehringer Ingelheim and has received lecture fees from Novo Nordisk, Eli Lilly, Sanofi, Boehringer Ingelheim, AstraZeneca, and Bristol-Myers Squibb. SS has occasionally been a consultant and received honorariums from Eli Lilly, Sanofi-Aventis, Novo Nordisk, Abbot Scandinavia, AstraZeneca, and Merck, Sharp \& Dohme and has participated in advisory boards for Sanofi-Aventis, AstraZeneca, and Eli Lilly. SD, SA, and N-GP has no such conflicts of interest to declare. Authors' spouses, partners, or children have no financial relationships that may be relevant to the submitted work; and no authors have non-financial interests that may be relevant to the submitted work. 
Ethical approval: This study was approved by the ethics committee of the University of Gothenburg, Gothenburg, Sweden (diary No 596-12).

Data sharing: The relevant anonymised patient level data are available on reasonable request from the corresponding author (lind. marcus@telia.com)

Transparency: The lead author (ML) affirms that the manuscript is an honest, accurate, and transparent account of the study being reported; that no important aspects of the study have been omitted; and that any discrepancies from the study as planned (and, if relevant, registered) have been explained.

This is an Open Access article distributed in accordance with the Creative Commons Attribution Non Commercial (CC BY-NC 4.0) license, which permits others to distribute, remix, adapt, build upon this work non-commercially, and license their derivative works on different terms, provided the original work is properly cited and the use is noncommercial. See: http://creativecommons.org/licenses/by-nc/4.0/.

1 UK Prospective Diabetes Study Group. Intensive blood-glucose control with sulphonylureas or insulin compared with conventional treatment and risk of complications in patients with type 2 diabetes (UKPDS 33). Lancet 1998:352:837-53

2 Holman RR, Paul SK, Bethel MA, Matthews DR, Neil HA. 10-year follow-up of intensive glucose control in type 2 diabetes. N Engl J Med 2008;359:1577-89.

3 Lind M, Olsson M, Rosengren A, Svensson AM, Bounias I, Gudbjörnsdottir S. The relationship between glycaemic control and heart failure in 83,021 patients with type 2 diabetes. Diabetologia 2012;55:2946-53.

4 American Diabetes Association. Standards of medical care in diabetes-2014. Diabetes Care 2014;37(Suppl 1):S14-80

5 Nationella riktlinjer för diabetesvård (National guidelines for diabetes care). National Board of Health and Welfare Sweden, 2015 www. socialstyrelsen.se/nationellariktlinjerfordiabetesvard.

6 Inzucchi SE, Bergenstal RM, Buse JB, et al. Management of hyperglycaemia in type 2 diabetes: a patient-centered approach. Position statement of the American Diabetes Association (ADA) and the European Association for the Study of Diabetes (EASD) Diabetologia 2012;55:1577-96.

7 Lind M, Pivodic A, Cea-Soriano L, Nerman O, Pehrsson NG, Garcia-Rodriguez LA. Changes in HbA1c and frequency of measuring $\mathrm{HbA} 1 \mathrm{C}$ and adjusting glucose-lowering medications in the 10 years following diagnosis of type 2 diabetes: a population-based study in the UK. Diabetologia 2014;57:1586-94

8 Inzucchi SE, Bergenstal RM, Buse JB, et al. Management of hyperglycemia in type 2 diabetes, 2015: a patient-centered approach: update to a position statement of the American Diabetes Association and the European Association for the Study of Diabetes. Diabetes Care 2015:38:140-9.

9 Pi-Sunyer FX. The impact of weight gain on motivation, compliance, and metabolic control in patients with type 2 diabetes mellitus. Postgrad Med 2009;121:94-107.

10 Lind M, Jendle J, Torffvit O, Lager I. Glucagon-like peptide 1 (GLP-1) analogue combined with insulin reduces $\mathrm{HbA} 1 \mathrm{c}$ and weight with low risk of hypoglycemia and high treatment satisfaction. Prim Care Diabetes 2012:6:41-6.

11 Lind M, Hirsch IB, Tuomilehto J, Dahlqvist S, Torffvit O, Pehrsson NG. Design and methods of a randomised double-blind trial of adding liraglutide to control $\mathrm{HbA} 1 \mathrm{c}$ in patients with type 2 diabetes with impaired glycaemic control treated with multiple daily insulin injections (MDI-Liraglutide trial). Prim Care Diabetes 2015;9:15-22.

12 Hirsch IB. Clinical review: realistic expectations and practical use of continuous glucose monitoring for the endocrinologist. J Clin Endocrinol Metab 2009;94:2232-8.

13 Workgroup on Hypoglycemia, American Diabetes Association. Defining and reporting hypoglycemia in diabetes: a report from the American Diabetes Association Workgroup on Hypoglycemia. Diabetes Care 2005;28:1245-9.

14 Lind M. Incretin therapy and its effect on body weight in patients with diabetes. Prim Care Diabetes 2012;6:187-91.

15 Ahrén B. Insulin plus incretin: a glucose-lowering strategy for type 2-diabetes. World J Diabetes 2014;5:40-51

16 Fonseca V, Schweizer A, Albrecht D, Baron MA, Chang I, Dejager S. Addition of vildagliptin to insulin improves glycaemic control in type 2 diabetes. Diabetologia 2007;50:1148-55.

17 Kothny W, Foley J, Kozlovski P, Shao Q, Gallwitz B, Lukashevich V. Improved glycaemic control with vildagliptin added to insulin, with or without metformin, in patients with type 2 diabetes mellitus. Diabetes Obes Metab 2013;15:252-7.

18 Vilsbøll T, RosenstockJ, Yki-Järvinen $\mathrm{H}$, et al. Efficacy and safety of sitagliptin when added to insulin therapy in patients with type 2 diabetes. Diabetes Obes Metab 2010;12:167-77.

19 Yki-Järvinen H, Rosenstock J, Durán-Garcia S, et al. Effects of adding linagliptin to basal insulin regimen for inadequately controlled type 2 diabetes: a $\geq 52$-week randomized, double-blind study. Diabetes Care 2013;36:3875-81
20 Buse JB, Bergenstal RM, Glass LC, et al. Use of twice-daily exenatide in basal insulin-treated patients with type 2 diabetes: a randomized, controlled trial. Ann Intern Med 2011:154:103-12.

21 Riddle MC, Aronson R, Home P, et al. Adding once-daily lixisenatide for type 2 diabetes inadequately controlled by established basal insulin: a 24-week, randomized, placebo controlled comparison (GetGoal-L). Diabetes Care 2013;36:2489-96.

22 Riddle MC, Forst T, Aronson R, et al. Adding once-daily lixisenatide for type 2 diabetes inadequately controlled with newly initiated and continuously titrated basal insulin glargine: a 24-week, randomized, placebocontrolled study (GetGoal-Duo 1). Diabetes Care 2013;36:2497-503.

23 Mathieu C, Rodbard HW, Cariou B, et al; BEGIN: VICTOZA ADD-ON (NN1250-3948) study group. A comparison of adding liraglutide versus a single daily dose of insulin aspart to insulin degludec in subjects with type 2 diabetes (BEGIN: VICTOZA ADD-ON). Diabetes Obes Metab 2014;16:636-44

24 Rosenstock J, Fonseca VA, Gross JL, et al; Harmony 6 Study Group. Advancing basal insulin replacement in type 2 diabetes inadequately controlled with insulin glargine plus oral agents: a comparison of adding albiglutide, a weekly GLP-1 receptor agonist, versus thrice-daily prandial insulin lispro. Diabetes Care 2014;37:2317-25.

25 Pratley RE, Nauck M, Bailey T, et al for 1860-LIRA-DPP-4 Study Group. Liraglutide versus sitagliptin for patients with type 2 diabetes who did not have adequate glycaemic control with metformin: a 26-week, randomised, parallel-group, open-label trial. Lancet 2010;375:1447-56

26 Buse JB, Rosenstock J, Sesti G, et al. Liraglutide once a day versus exenatide twice a day for type 2 diabetes: a 26-week randomised, parallel-group, multinational, open-label trial (LEAD-6). Lancet 2009;374:39-47.

27 Buse JB, Nauck M, Forst T, et al. Exenatide once weekly versus liraglutide once daily in patients with type 2 diabetes (DURATION-6): a randomised, open-label study. Lancet 2013;381:117-24.

28 Fujiwara D, Takahashi K, Suzuki T, et al. Postprandial serum C-peptide value is the optimal index to identify patients with non-obese type 2 diabetes who require multiple daily insulin injection: analysis of $\mathrm{C}$-peptide values before and after short-term intensive insulin therapy. I Diabetes Investig 2013:4:618-25.

29 Kahn SE. Clinical review 135: the importance of beta-cell failure in the development and progression of type 2 diabetes. J Clin Endocrinol Metab 2001;86:4047-58

30 Action to Control Cardiovascular Risk in Diabetes Study Group, Gerstein HC, Miller ME, Byington RP, et al. Effects of intensive glucose lowering in type 2 diabetes. N Engl J Med 2008;358:2545-59.

31 ADVANCE Collaborative Group, Patel A, MacMahon S, Chalmers J, et al. Intensive blood glucose control and vascular outcomes in patients with type 2 diabetes. N Engl / Med 2008;358:2560-72.

32 Schauer PR, Kashyap SR, Wolski K, et al. Bariatric surgery versus intensive medical therapy in obese patients with diabetes. N EnglJ Med 2012;366:1567-76.

33 Rosenstock J, Fonseca V. Missing the point: substituting exenatide for nonoptimized insulin: going from bad to worse! Diabetes Care 2007;30:2972-3

34 UK Prospective Diabetes Study Group. Tight blood pressure control and risk of macrovascular and microvascular complications in type 2 diabetes: UKPDS 38. BM/ 1998:317:703-13

35 Garber A, Henry RR, Ratner R, Hale P, Chang CT, Bode B; LEAD-3 (Mono) Study Group. Liraglutide, a once-daily human glucagon-like peptide 1 analogue, provides sustained improvements in glycaemic control and weight for 2 years as monotherapy compared with glimepiride in patients with type 2 diabetes. Diabetes Obes Metab 2011;13:348-56.

36 Marso SP, Lindsey JB, Stolker JM, et al. Cardiovascular safety of liraglutide assessed in a patient-level pooled analysis of phase 2:3 liraglutide clinical development studies. Diab Vasc Dis Res 2011;8:237-40.

37 Marso SP, Poulter NR, Nissen SE, et al. Design of the liraglutide effect and action in diabetes: evaluation of cardiovascular outcome results (LEADER) trial. Am Heart J 2013;166:823-30.e5

38 Pfeffer MA, Diaz R, Lewis E, et al. The evaluation of lixisenatide in acute coronary syndrome -the results of the ELIXA trial. Symposium at the 75th Scientific Sessions of the American Diabetes Association; Boston, MA; 8 Jun, 2015.

39 Robinson LE, Holt TA, Rees K, Randeva HS, O'Hare JP. Effects of exenatide and liraglutide on heart rate, blood pressure and body weight: systematic review and meta-analysis. BMJ Open 2013;3(1):pii:e001986.

40 Egan AG, Blind E, Dunder K, et al. Pancreatic safety of incretin-based drugs-FDA and EMA assessment. N Engl J Med 2014;370:794-7.

41 Hegedüs L, Moses AC, Zdravkovic M, Le Thi T, Daniels GH. GLP-1 and calcitonin concentration in humans: lack of evidence of calcitonin release from sequential screening in over 5000 subjects with type 2 diabetes or nondiabetic obese subjects treated with the human GLP-1 analog, liraglutide. J Clin Endocrinol Metab 2011;96:853-60.

(c) BMJ Publishing Group Ltd 2015

Web material: supplementary appendix 